\title{
NEAR-INFRARED IMAGING WITH A SCHMIDT TELESCOPE
}

\author{
K. YANAGISAWA ${ }^{1}$, N. ITOH ${ }^{1}$, T. ICHIKAWA ${ }^{2}$, K. TARUSAWA ${ }^{2}$ and M. UENO ${ }^{3}$ \\ ${ }^{1}$ Department of Astronomy, University of Tokyo, Yayoi, Bunkyo-ku, Tokyo 113, \\ Japan \\ ${ }^{2}$ Kiso Observatory, University of Tokyo, Mitake-Mura, Kiso-gun, Nagano 397-01, \\ Japan \\ ${ }^{3}$ College of Arts and Science, University of Tokyo, Komaba, Meguro-ku, Tokyo 253, \\ Japan
}

\begin{abstract}
We have carried out wide field imaging observations in the near-infrared $\left(J, H\right.$ and $K^{\prime}$ band) with a large format array camera attached to the prime focus of the $105 \mathrm{~cm}$ Schmidt telescope at Kiso Observatory. The image resolution, limiting magnitudes and the effect of thermal radiation are discussed.
\end{abstract}

CCD cameras with a large format array and mosaic CCDs have replaced photographic plates as powerful detectors for observations at optical wavelengths. Wide-field imaging in near-infrared bands, which is important for studies of nearby galaxies and star forming regions in our Galaxy, has not been performed yet, since neither plates nor large format arrays sensitive at wavelengths longer than $1 \mu \mathrm{m}$ are available. The $256 \times 256 \mathrm{HgCdTe}$ array is currently the most popular and largest array in the near-infrared. These arrays may be, however, too small for wide-field imaging.

We have tested a unique combination of an infrared camera with a large format array (Ueno et al. 1992) and a fast f-ratio telescope. The detector used is a Platinum Silicide Schottky barrier diode array (Kimata et al. 1987) supplied by Mitsubishi Electric Co. The array size is $512 \times 512$ pixels, and the pixel size is $26 \mu \mathrm{m} \times 20 \mu \mathrm{m}$. The field-of-view is $14^{\prime} \times 11^{\prime}$ and the pixel resolution is $1.6^{\prime \prime} \times 1.3^{\prime \prime}$ when attached to the prime focus of the $105 \mathrm{~cm}$ Schmidt telescope at Kiso Observatory.

Though the quantum efficiency of the array is low, the excellent uniformity, stability and low read-out noise of the camera combined with the fast $f$-ratio of the telescope resulted in a calibration accuracy of $0.1 \%$ of the sky background in an image obtained through 70 minute exposure in the $H$-band. The observationally confirmed detection limits are shown in Table 1.

The thermal radiation from the Schmidt telescope is very large in the $K^{\prime}$ band as shown in Fig. 1. Though the camera does not have a Lyot-stop to reduce the thermal emission from inside the telescope, it was confirmed to be negligible in the $J$ and $H$ bands. The plots shown in Fig. 1 are based on model calculations, which were found to be consistent with observations.

We also carried out a Hartmann test to evaluate the image sharpness of the Kiso Schmidt telescope in near-infrared bands. The Hartmann constants $(T)$ are $T=0.98$ and $1.12 \operatorname{arcsec}$ for $J$ and $H$ band, respectively. The analysis for the $K^{\prime}$ band is in progress. The seeing size at Kiso is typically about 4 arcsec. We can therefore conclude that seeing limited images can be taken, 


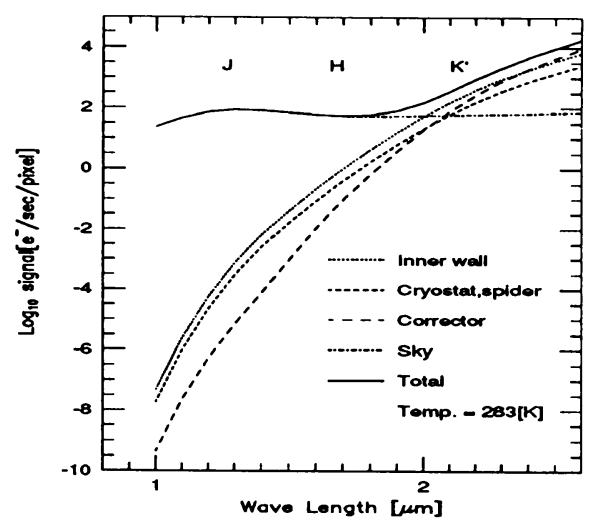

Figure 1. Thermal radiation from the inside of the Kiso Schmidt telescope.

taking the Hartmann constants and the pixel resolution into consideration.

A camera with PtSi $1040 \times 1040$ chip is now under development, using a chip with an improved quantum efficiency and filling factor. The field-of-view will be $18^{\prime} \times 18^{\prime}$ at prime focus of the Kiso Schmidt telescope. It will be a powerful tool for imaging objects of large apparent size and for surveys. We are planning to obtain multi-colour photometry at optical and infrared wavelengths of nearby galaxies.

Table 1. Detection limit $(\mathrm{S} / \mathrm{N}=1.0$, exp $=20 \mathrm{~min}$, seeing $=4 \operatorname{arcsec})$

\begin{tabular}{ccc}
\hline Band & for Diffuse Objects & for Point Objects \\
\hline \hline$J(1.25 \mu \mathrm{m})$ & $21.5 \mathrm{mag} / \operatorname{arcsec}^{2}$ & $20.5 \mathrm{mag}$ \\
$H(1.65 \mu \mathrm{m})$ & $19.6 \mathrm{mag} / \operatorname{arcsec}^{2}$ & $18.6 \mathrm{mag}$ \\
$K^{\prime}(2.15 \mu \mathrm{m})$ & $17.6 \mathrm{mag} / \operatorname{arcsec}^{2}$ & $16.5 \mathrm{mag}$ \\
\hline
\end{tabular}

\section{References}

Kimata, M. et al., 1987. IEEE Journal of Solid State Circuits, sc-22 6, 1124.

Ueno, M. et al., 1992. S.P.I.E., 1762, 423. 